\title{
Extracción endovascular de cuerpo extraño intracardiaco posterior a fractura y embolización de catéter venoso: reporte de un caso
}

\author{
Endovascular extraction of intracardiac foreign body posterior to fracture and \\ embolization of venous catheter: a case report
}
Extração endovascular de corpo estranho intracardíaco após fratura e embolização por cateter venoso: relato de caso

\section{Leonardo Matute Rivera (D)a, Diana Núñez Parra (D) b, Luciana Boloña Gilbert (D) c, Jorge Andrade Pacheco iD $^{d}$}

\begin{abstract}
a,b,c Servicio de Cirugía General, Hospital Clínica Guayaquil, Guayaquil, Ecuador; d Servicio de Cardiología Intervencionista, Hospital Clínica Guayaquil, Guayaquil, Ecuador.
\end{abstract}

\section{Correspondencia a:}

Leonardo Matute Rivera,

lixp05@hotmail.com

Recibido: 14 de octubre, 2020

Aceptado: 15 de noviembre, 2020

Publicado: 30 de diciembre, 2020

\section{CASO CLÍNICO}

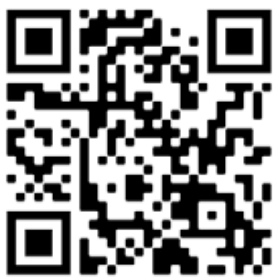

Escanea en tu dispositivo móvil o revisa este artículo en: https:// revistaclinicaguayaquil.org

\section{RESUMEN}

El uso de puertos venosos implantables tiene un auge importante, especialmente en pacientes oncológicos. Dichos puertos tienen múltiples usos, fundamentalmente para la administración de quimioterapia. El presente artículo trata de una paciente femenina de 51 años de edad, con diagnóstico de cáncer de mama con requerimiento de quimioterapia, cuyo implante intravenoso se fractura y un fragmento del mismo migra hacia las cavidades cardiacas; para su tratamiento se realiza la extracción del catéter de forma endovascular.

Palabras clave: catéter venoso; embolización; extracción endovascular

\begin{abstract}
The use of implantable venous ports has had a significant increase, especially in cancer patients. These ports have multiple uses, mainly for the administration of chemotherapy. This article describes a 51-year-old female patient, diagnosed with breast cancer requiring chemotherapy, whose intravenous implant fractures and a fragment of it migrates into the cardiac cavities; for its treatment, the catheter is extracted endovascularly
\end{abstract}

Key words: venous catheter; embolization; endovascular extraction

\section{RESUMO}

O uso de portos venosos implantáveis tem um boom significativo, especialmente em pacientes com câncer. Esses portos têm usos múltiplos, principalmente para a administração da quimioterapia. Este artigo é sobre uma paciente mulher, de 51 anos de idade, paciente diagnosticada com câncer de mama com exigência de quimioterapia, cujo implante intravenoso, fratura-se, e um fragmento dele migra para as câmaras cardíacas; pelo qual é desenvolvido o tratamento baseado numa remoção do cateter, ou seja, uma extração endo vascular.

Palavras-chave: cateter venoso; embolização; extração endovascular

\section{INTRODUCCIÓN}

El uso de puertos intravenosos implantables data desde 1929, ya para 1979 se usó un catéter de Hickman para la administración de quimioterapia, y para 1980 se implantan puertos intravenosos 
subcutáneos, proporcionando una vía segura para la administración intravenosa de medicamentos por periodos prolongados de tiempo $(1,2)$.

Los catéteres venosos con reservorio subcutáneo constan de dos componentes: el portal o reservorio, que posee un septum de silicona que permite su acceso por punción percutánea, y el catéter radiopaco (2). Su uso ha aumentado los últimos años, manteniendo accesos venosos por largo tiempo, pero también presentan riesgo de complicaciones destacando las infecciosas, complicaciones trombóticas, migración del catéter, oclusión, neumotórax al momento del implante y las erosiones vasculares (3).

\section{DESCRIPCIÓN DEL CASO CLÍNICO}

Se presenta el caso de una paciente femenina de 51 años de edad, con antecedentes de cáncer de mama izquierda con tratamiento quirúrgico con mastectomía radical izquierda y posteriormente se indicó tratamiento con quimioterapia, por lo que se coloca un catéter de reservorio subcutáneo (Celsite ${ }^{\circledR}$ Implantofix ${ }^{\circledR}$ Access Port) por vía subclavia izquierda. Tres meses después de la colocación del dispositivo presenta disfunción del catéter, presentando dolor durante infusión de medicación, y obstrucción del flujo. A la exploración física, no se evidenciaron signos de inflamación en el área del bolsillo del catéter. Se realizó una radiografía de tórax de control, en la que se observó el reservorio sobre la parrilla costal izquierda y una solución de continuidad correspondiente a la sección del catéter a nivel del espacio costoclavicular; además, se localizó el extremo distal del catéter en cavidades cardiacas derechas y tronco de arteria pulmonar. Posteriormente, se realiza una tomografía computarizada simple de tórax y se confirman hallazgos (Figura 1).

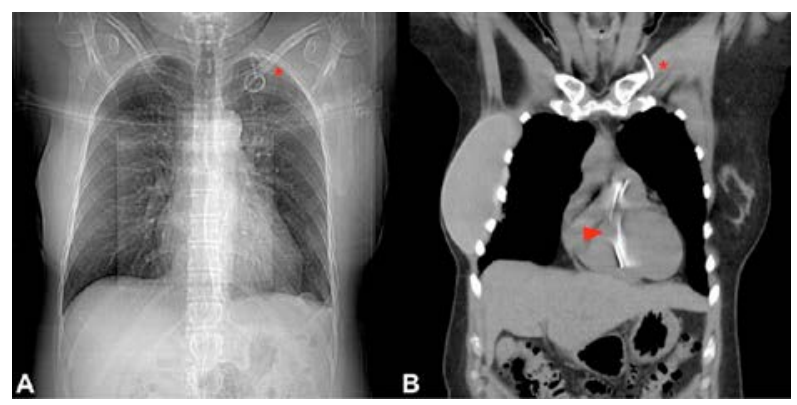

Figura 1. A. Topograma de tomografía computarizada, se evidencia extremo del catéter amputado a nivel proximal (*). B. Tomografía computarizada de tórax, corte coronal, se observa el reservorio y el extremo del catéter proximal $\left({ }^{*}\right)$ y extremo distal en cavidades derechas (ver punta de flecha).
Una vez confirmado el diagnóstico, los servicios de Cirugía y de Hemodinámica proceden al retiro del puerto subcutáneo. El procedimiento se lleva a cabo con anestésico local, y luego con sedación; se procede por acceso femoral derecho, y con la ayuda de catéter de lazo (Snare), se realiza el retiro del fragmento del catéter de las cavidades cardiacas (Figura 2). A las 48 horas del retiro del catéter se procede a colocar un nuevo catéter de reservorio subcutáneo (Celsite ${ }^{\circledR}$ Implantofix ${ }^{\circledR}$ Access Port) por vía yugular derecha. A I día siguiente la paciente es dada de alta en condiciones clínicas estables.

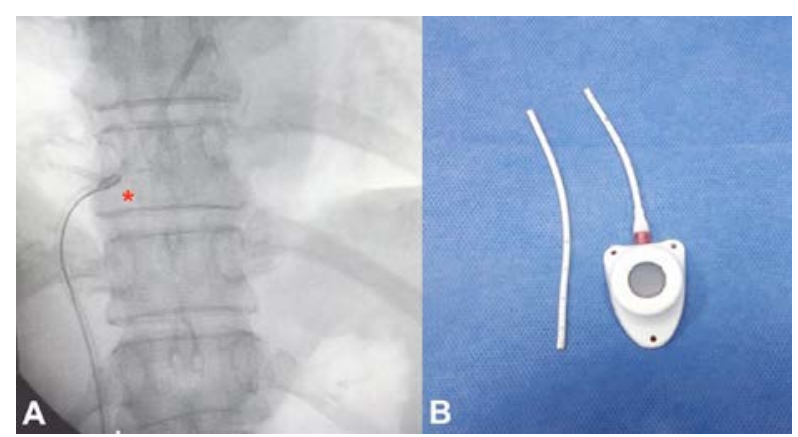

Figura 2. A. Radiología donde se observa extracción del extremo distal del catéter mediante el uso de un catéter de lazo (Snare) (*). B. Se observa el fragmento distal y el reservorio.

\section{DISCUSIÓN}

Con el aumento del uso de dispositivos de reservorio subcutáneo para mantener un acceso vascular de larga duración, existen documentados casos de fractura espontánea del extremo distal del catéter, con embolización del mismo. El reservorio se coloca sobre la parrilla costal, y la proximidad a estructuras óseas, puede ocasionar desgaste y fractura del catéter. La embolización del extremo distal del catéter es más frecuente hacia cavidades cardiacas derechas y a la arteria pulmonar; esta puede traer serias complicaciones, siendo las más frecuentes las arritmias cardiacas (4). El diagnóstico de rotura del catéter del reservorio se lo realiza a través de la sospecha clínica y apoyado con medios radiológicos, en este caso la tomografía computarizada de tórax evidencia segmento distal de catéter ubicado en ventrículo derecho y arteria pulmonar (3).

Los síntomas más frecuentes incluyen dolor del área del reservorioylasensación de palpitaciones, aunque según la literatura, algunos pacientes pueden cursarla de forma asintomática $(3,4)$. En el caso en desarrollo, la paciente presentó dolor y disfunción del dispositivo. Una vez confirmado el diagnóstico, se requiere el retiro del puerto 
subcutáneo y del catéter, ya sea por cirugía o de forma endovascular (3).

\section{REFERENCIAS BIBLIOGRÁFICAS}

1. Teichgräber RU, Pfitzmann R, Hofmann H. Los sistemas de puertos venosos centrales como parte integral de la quimioterapia. Dtsch Ärzteblatt Int. 2011;108:147-54

2. Vázquez Luna F, Jordán González JA. Colocación de reservorio subcutáneo venoso central permanente. Investig Medicoquirúrgicas. 2018;10(1):167-79

3. Vidal MA, García-Hernández R, Torres LM. Rotura espontánea de catéter venoso central. Rev la Soc Española del Dolor. 2006;13(3):164-6

4. Espinoza-Contreras C, Valverde-Muñoz K. Migración de fragmentos de catéteres percutáneos en paciente pediátrico. Acta Med Costarric. 2017;59(4):167-9

\section{ACERCA DE LOS AUTORES}

1. Leonardo Matute Rivera: Cirujano General, Universidad de Especialidades Espíritu Santo. Servicio de Cirugía General, Hospital Clínica Guayaquil, Guayaquil, Ecuador.

\section{ORCID: 0000-0002-6867-0700}

2. Diana Núñez Parra: Médico Posgradista de Cirugía General, Universidad de Especialidades Espíritu Santo, Hospital Clínica Guayaquil; Guayaquil, Ecuador.

ORCID: 0000-0002-2978-9311

3. Luciana Boloña Gilbert: Cirujano General, Universidad de Especialidades Espíritu Santo. Servicio de Cirugía General, Hospital Clínica Guayaquil, Guayaquil, Ecuador.

ORCID: 0000-0002-0319-9882

4. Jorge Andrade Pacheco: Cardiólogo intervencionista especializado en el Hospital Álvaro Cunqueiro. Cardiólogo especializado en la Universidad Favaloro/Fundación Favaloro. Servicio de Cardiología Intervencionista, Hospital Clínica Guayaquil, Guayaquil, Ecuador.

ORCID: 0000-0002-7827-2925 